Original Research Paper

\title{
Interaction Model in Statistical Mechanics
}

\author{
${ }^{1}$ Farida Kachapova and ${ }^{2}$ Ilias Kachapov \\ ${ }^{1}$ Auckland University of Technology, New Zealand \\ ${ }^{2}$ University of Auckland, New Zealand
}

Article history

Received: 28-08-2017

Revised: 30-08-2017

Accepted: 03-11-2017

Corresponding Author:

Farida Kachapova

Department of Mathematical

Sciences,

Auckland University of

Technology, New Zealand

Email: farida.kachapova@aut.ac.nz

\begin{abstract}
Statistical mechanics considers several models such as Ising model, Potts model, Heisenberg model etc. A rigorous mathematical approach based on the axiomatic foundation of probability would benefit the study and applications of these models. In this paper we use this approach to generalize some of these models into one construction named an interaction model. We introduce a mathematically rigorous definition of the model on an integer lattice that describes a physical system with many particles interacting with an external force and with one another; a random field $X_{t}\left(t \in \mathbb{Z}^{v}\right)$ models some property of the system such as electric charge, density etc. We introduce a finite model first and then define the thermodynamic limit of the finite models with Gibbs probability measure. The set of values of $X_{t}$ can be unbounded for more generality. We study properties of the interaction model and show that Ising and Potts models are particular cases of the interaction model.
\end{abstract}

Keywords: Infinite Particle System, Gibbs Measure, Radius of Interaction, Thermodynamic Limit, Ising Model, Potts Model

\section{Introduction}

Statistical mechanics studies models of physical systems with many particles, which interact with an external force and with one another. Well-known models include Ising, Potts, Heisenberg, and $n$-vector models (see, for example, Duminil-Copin et al., 2017; Kashapov, 1977; Külske et al., 2014; Malyshev and Minlos, 1991; Malyshev, 1980). In these models a random field $X_{t}$ is used to model some property of the system such as electric charge, density etc.

Ising model is the simplest and most popular model. It describes a system with two states and models the phenomenon of ferromagnetism. It is also used in quantum field theory. Potts model is a generalization of Ising model to a system with a finite number of states.

An $n$-vector model represents classical spins by $n$ dimensional vectors of unit length. This model can be used to describe many physical phenomena. Particular cases of this model include the Ising model for $n=1$, $X Y$-model for $n=2$ and Heisenberg model for $n=3$.

Kachapova and Kachapov (2016) introduced the concept of interaction model as a generalization of some existing models; there we provided a proof based on this concept that the random field $X_{t}$ transformed by renormalization group converges to an independent random field with Gaussian distribution.
In this paper we generalize and improve the interaction model from (Kachapova and Kachapov, 2016). The new model does not have restrictions on the distribution of $X_{t}$ and the set of values of $X_{t}$ can be unbounded, which is an advantage of this model comparing to all aforementioned models, which have the values of $X_{t}$ bounded.

In this paper we use a rigorous mathematical approach based on the axiomatic foundation of probability. We introduce a mathematically precise definition of interaction model on an integer lattice: first as a finite model and then as the thermodynamic limit of the finite models with Gibbs probability measure.

We study properties of the interaction model and show how some well-known models are represented as particular cases of the interaction model.

In Section 1 we introduce main components of the interaction model of a physical system that include an integer lattice $\mathbb{Z}^{v}$, the set of configurations of the system, initial independent probability measure $P_{0}$ and a random field $X_{t}\left(t \in \mathbb{Z}^{v}\right)$ that models a property of the system. Next we introduce three characteristics of the interaction model: a main parameter $\lambda$, radius of interaction $r$ and potential $\Phi$.

In Section 2 we study Gibbs modification of a probability measure. In particular, we split Gibbs 
modification of the initial independent probability measure into two steps reflecting the influence of an external field on the first step and the interaction between particles on the second step. The first-step modification is mathematically simple and leaves the field $X_{t}$ independent, therefore this construction simplifies mathematical computations.

In Section 3 we define a finite interaction model on an integer cube using Gibbs modification of the initial probability measure $P_{0}$. We prove some properties of the finite model.

In Section 4 we define an infinite interaction model and in Section 5 we show that Ising and Potts models are particular cases of the interaction model. In Section 6 we discuss how to generalize our model, so that the $n$-vector model becomes its particular case too.

\section{Main Components of Interaction Model}

In Section 3 we will construct an interaction model to describe a physical system with many particles. Here we introduce its main components.

\section{Definition 1.1}

1. Fix a natural number $v \geq 1$ and consider a $v$ dimensional integer lattice:

$$
\mathbb{Z}^{v}=\left\{\left(\tau_{1}, \ldots, \tau_{2}\right) \mid \tau_{i} \in \mathbb{Z}, i=1, \ldots, v\right\}
$$

with the distance between any two points $s, \tau \in \mathbb{Z}^{v}$ defined by:

$$
\|S-\tau\|=\sum_{i=1}^{v}\left|s_{i}-\tau_{i}\right|
$$

2. $\Omega=\left\{\omega \mid \omega: \mathbb{Z}^{v} \rightarrow \mathbb{R}\right\}$.

An element $\omega$ of $\Omega$ is called a configuration and is interpreted as a state of the physical system

3. For any $t \in \mathbb{Z}^{v}$ a function $X_{t}: \Omega \rightarrow \mathbb{R}$ is defined by the following:

$$
X_{t}(\omega)=\omega(t)
$$

4. Denote $\Sigma$ the $\sigma$-algebra generated by sets of the form $\{\omega \in \Omega \mid \omega(t) \leq a\}$ for all $t \in \mathbb{Z}^{v}, a \in \mathbb{R}$.

5. Fix $P_{0}$, a probability measure on $(\Omega, \Sigma)$ such that for any $a_{1}, \ldots, a_{n} \in \mathbb{R}$ and distinct $t_{1}, \ldots, t_{n} \in \mathbb{Z}^{v}$ :

$$
P_{0}\left(\bigcap_{i=1}^{n}\left\{\omega\left(t_{i}\right) \leq a_{i}\right\}\right)=\prod_{i=1}^{n} P_{0}\left(\omega\left(t_{i}\right) \leq a_{i}\right) .
$$

We call $P_{0}$ the initial probability measure.
For the rest of the paper we fix the objects $\mathbb{Z}^{v}, \Omega, X, \Sigma$ and $P_{0}$ from this definition.

\section{Remark}

There always exists $P_{0}$ satisfiying (1). For example, if $F$ is any probability distribution function, we can take $P_{0}(\omega(t) \leq a)=F(a)$ for any $t$ and define the rest by formula (1).

\section{Lemma 1.2}

1) $\left(\Omega, \Sigma, P_{0}\right)$ is a probability space.

2) $\left\{X_{t} \mid t \in \mathbb{Z}^{v}\right\}$ is an independent random field on this probability space.

\section{Proof}

The lemma immediately follows from the definitions.

\section{Definition 1.3}

1. Consider a graph $(V, E)$, where the set of vertices $V$ is a finite subset of $\mathbb{Z}^{v}$ and $E$ is the set of edges; each edge can be regarded as a pair of distinct vertices (there are no loops). The length of each edge is the distance between its end vertices.

2. The graph $(V, E)$ is called 1-connected if it is connected and the length of any of its edges equals 1.

3. For a finite set $B \subset \mathbb{Z}^{v}$ define its size $S(B)$ as the minimum number of edges of 1-connected graphs $(V, E)$ such that $B \subseteq V$.

\section{Definition 1.4}

Here we introduce three main characteristics of interaction: $\lambda, r, \Phi$ and a set $\mathfrak{B}$.

1. $\lambda \in \mathbb{R}, 0 \leq \lambda<1$. $\lambda$ is called the main parameter of the model.

2. $r \in \mathbb{R}, r \geq 1$. $r$ is called the radius of interaction.

3. Denote $\mathfrak{B}=\left\{B \subset \mathbb{Z}^{v} \mid B \neq \varnothing\right.$ and size $\left.S(B) \leq r\right\}$.

4. For each $B \in \mathfrak{B}, \Phi_{B}$ is a random variable on the probability space $\left(\Omega, \Sigma_{B}, P_{0}\right)$, where $\sum_{B}$ is the $\sigma$ algebra generated by sets of the form $\{\omega \in \Omega \mid \omega(t) \leq a\}, t \in B, a \in \mathbb{R} ; \quad$ all $\Phi_{B}$ satisfy the condition:

$$
\left|\Phi_{B}\right| \leq \lambda^{S(B)} .
$$

$\Phi$ is called the potential of the system. 
Clearly, each set $B \in \mathfrak{B}$ is finite. $\Phi_{B}$ characterizes the interaction energy of the set $B$. If $B$ consists of two or more points, then the random variable $\Phi_{B}$ represents interaction between elements of the set $B$. If $B=\{t\}$ is a singleton, then $\Phi_{B}$ represents interaction of $t$ with an external field and the influence of kinetic energy.

\section{Lemma 1.5}

If sets $B, C \in \mathfrak{B}$ and $B \cap C=\varnothing$, then the random variables $\Phi_{B}$ and $\Phi_{C}$ are independent with respect to the probability measure $P_{0}$, that is on the probability space $\left(\Omega, \Sigma, P_{0}\right)$.

\section{Proof}

This is proven using standard techniques of probability theory, first for the case of discrete $\Phi_{B}$ and $\Phi_{C}$, and then for general random variables $\Phi_{B}, \Phi_{C}$ as limits of discrete random variables.

\section{Gibbs Modification}

To describe interaction between particles, we modify the initial probability measure $P_{0}$, so that the corresponding distribution of the random field $\left\{X_{t} \mid t \in \mathbb{Z}^{v}\right\}$ is not independent any more. This section describes the modification in general.

For any probability measure $P$ on $(\Omega, \Sigma)$ denote $\langle\cdot\rangle_{P}$ the expectation with respect to $P$.

\section{Definition 2.1}

Suppose $P$ is a probability measure on $(\Omega, \Sigma)$ and $U$ is a bounded random variable on $(\Omega, \Sigma)$.

Gibbs modification of the probability measure $P$ by the random variable $U$ is denoted $P_{U}$ and is defined as follows. For any event $A \in \Sigma$ :

$$
P_{U}(A)=\frac{\left\langle I_{A} e^{U}\right\rangle_{P}}{\left\langle e^{U}\right\rangle_{P}}
$$

where $I_{A}$ denotes the indicator of event $A$.

\section{Remark}

Since the random variable $U$ is bounded, both expectations in formula (2) exist and $\left\langle e^{U}\right\rangle_{P}>0$. So $P_{U}(A)$ is always defined.

The following lemma is used in literature without proof. In order to have a complete picture we provide an accurate proof here.

\section{Lemma 2.2}

In conditions of the previous definition:
1) $P_{U}$ is a probability measure on $(\Omega, \Sigma)$;

2) for any random variable $Y$ on $(\Omega, \Sigma),\langle Y\rangle_{P_{U}}=\frac{\left\langle e^{U}\right\rangle_{P}}{\left\langle e^{U}\right\rangle_{P}}$.

\section{Proof}

1)

$$
\begin{aligned}
& P_{U}(\varnothing)=\frac{\left\langle I_{\varnothing} e^{U}\right\rangle_{P}}{\left\langle e^{U}\right\rangle_{P}}=\frac{0}{\left\langle e^{U}\right\rangle_{P}}=0 ; \\
& P_{U}(\Omega)=\frac{\left\langle I_{\Omega} e^{U}\right\rangle_{P}}{\left\langle e^{U}\right\rangle_{P}}=\frac{\left\langle e^{U}\right\rangle_{P}}{\left\langle e^{U}\right\rangle_{P}}=1 .
\end{aligned}
$$

To complete the proof it remains to show that for any sequence of disjoint events $A_{i} \in \Sigma(i=1,2, \ldots)$ the following holds:

$P_{U}\left(\bigcup_{i=1}^{\infty} A_{i}\right)=\sum_{i=1}^{\infty} P_{U}\left(A_{i}\right)$.

Denote $A=\bigcup_{i=1}^{\infty} A_{i}$. First we prove:

$\left\langle I_{A} e^{U}\right\rangle_{P}=\sum_{i=1}^{\infty}\left\langle I_{A_{i}} e^{U}\right\rangle_{P}$.

$U$ is a bounded random variable, so for some constant $M,|U| \leq M$ and $0<e^{U} \leq e^{M}$.

Since $P$ is a probability measure, we have:

$$
P(A)=P\left(\bigcup_{i=1}^{\infty} A_{i}\right)=\sum_{i=1}^{\infty} P\left(A_{i}\right) .
$$

So for any $\varepsilon>0$ there is $n \in \mathbb{N}$ such that $\sum_{i=n+1}^{\infty} P\left(A_{i}\right) \leq \varepsilon e^{-M}$. Denote $B_{n}=\bigcup_{i=n+1}^{\infty} A_{i}$. Then:

$$
\begin{gathered}
P\left(B_{n}\right) \leq \varepsilon e^{-M} . \\
A=\bigcup_{i=1}^{n} A_{i} \cup B_{n}, \quad I_{A}=\sum_{i=1}^{n} I_{A_{i}}+I_{B_{n}} . \\
\left\langle I_{A} e^{U}\right\rangle_{P}=\left\langle\left(\sum_{i=1}^{n} I_{A_{i}}+I_{B_{n}}\right) e^{U}\right\rangle_{P}=\left\langle\sum_{i=1}^{n} I_{A_{i}} e^{U}+I_{B_{n}} e^{U}\right\rangle_{P} \\
=\sum_{i=1}^{n}\left\langle I_{A_{i}} e^{U}\right\rangle_{P}+\left\langle I_{B_{n}} e^{U}\right\rangle_{P} . \\
0 \leq\left\langle I_{A} e^{U}\right\rangle_{P}-\sum_{i=1}^{n}\left\langle I_{A_{i}} e^{U}\right\rangle_{P}=\left\langle I_{B_{n}} e^{U}\right\rangle_{P} \\
\leq\left\langle I_{B_{n}} e^{M}\right\rangle_{P} \leq e^{M}\left\langle I_{B_{n}}\right\rangle_{P}=e^{M} P\left(B_{n}\right) \\
\\
{[b y(5)] \leq e^{M} \varepsilon e^{-M}=\varepsilon .}
\end{gathered}
$$

Thus: 


$$
\left|\sum_{i=1}^{n}\left\langle I_{A_{i}} e^{U}\right\rangle_{P}-\left\langle I_{A} e^{U}\right\rangle_{P}\right| \leq \varepsilon
$$

Therefore $\lim _{n \rightarrow \infty} \sum_{i=1}^{n}\left\langle I_{A_{i}} e^{U}\right\rangle_{P}=\left\langle I_{A} e^{U}\right\rangle_{P}$. That is, the series $\sum_{i=1}^{\infty}\left\langle I_{A_{i}} e^{U}\right\rangle_{P}$ converges and $\sum_{i=1}^{\infty}\left\langle I_{A_{i}} e^{U}\right\rangle_{P}=\left\langle I_{A} e^{U}\right\rangle_{P}$. proven.

Proof of (3)

$$
\begin{aligned}
P_{U}\left(\bigcup_{i=1}^{\infty} A_{i}\right) & =P_{U}(A)=\frac{\left\langle I_{A} e^{U}\right\rangle_{P}}{\left\langle e^{U}\right\rangle_{P}}=[b y(4)]=\frac{\sum_{i=1}^{\infty}\left\langle I_{A_{i}} e^{U}\right\rangle_{P}}{\left\langle e^{U}\right\rangle_{P}} \\
& =\sum_{i=1}^{\infty} \frac{\left\langle I_{A_{i}} e^{U}\right\rangle_{P}}{\left\langle e^{U}\right\rangle_{P}}=\sum_{i=1}^{\infty} P_{U}\left(A_{i}\right) .
\end{aligned}
$$

2) Consider 3 cases.

- Case 1: $Y=I_{A}(Y$ is an indicator of some event $A \in \Sigma)$

Then $\langle Y\rangle_{P_{U}}=\left\langle I_{A}\right\rangle_{P_{U}}=P_{U}(A)=\frac{\left\langle I_{A} e^{U}\right\rangle_{P}}{\left\langle e^{U}\right\rangle_{P}}=\frac{\left\langle Y e^{U}\right\rangle_{P}}{\left\langle e^{U}\right\rangle_{P}}$.

- Case 2: $Y=\sum_{i=1}^{n} a_{i} Y_{i}$, where each $Y_{i}$ is an indicator.

Then:

$$
\begin{aligned}
\langle Y\rangle_{P_{U}} & =\sum_{i=1}^{n} a_{i}\left\langle Y_{i}\right\rangle_{P_{U}}=[\text { by Case 1] } \\
& =\sum_{i=1}^{n} a_{i} \frac{\left\langle Y_{i} e^{U}\right\rangle_{P}}{\left\langle e^{U}\right\rangle_{P}}=\frac{\sum_{i=1}^{n} a_{i}\left\langle Y_{i} e^{U}\right\rangle_{P}}{\left\langle e^{U}\right\rangle_{P}} \\
& =\frac{\left\langle\sum_{i=1}^{n} \alpha_{i} Y_{i} e^{U}\right\rangle_{P}}{\left\langle e^{U}\right\rangle_{P}}=\frac{\left\langle Y e^{U}\right\rangle_{P}}{\left\langle e^{U}\right\rangle_{P}} .
\end{aligned}
$$

- Case 3: general case.

It is a well known fact in probability theory that any random variable can be represented as a uniform limit of discrete random variables. Thus, for any $\omega \in \Omega$ :

$$
Y(\omega)=\lim _{n \rightarrow \infty} V_{n}(\omega),
$$

where the convergence is uniform with respect to $\omega \in \Omega$ and each $V_{n}$ is a discrete random variable with a finite number of values; this is a random variable from Case 2.

Then:

$$
\lim _{n \rightarrow \infty}\left\langle V_{n}\right\rangle_{P_{U}}=\langle Y\rangle_{P_{U}}
$$

As $n \rightarrow \infty$, we have for any $\omega \in \Omega: V_{n}(\omega)-Y(\omega) \rightarrow 0$ uniformly on $\Omega$, so $\left|V_{n}(\omega)-Y(\omega)\right| \rightarrow 0$ and:

$$
\lim _{n \rightarrow \infty}\left\langle\left|V_{n}-Y\right|\right\rangle_{P} \rightarrow 0 \text { as } n \rightarrow \infty \text {. }
$$

$U$ is a bounded random variable, so for some constant $M,|U| \leq M$ and $0<e^{U} \leq e^{M}$.

$$
\begin{aligned}
& \left|\left\langle V_{n} e^{U}\right\rangle_{P}-\left\langle Y e^{U}\right\rangle_{P}\right|=\left|\left\langle\left(V_{n}-Y\right) e^{U}\right\rangle_{P}\right| \\
& \leq\left\langle\left|\left(V_{n}-Y\right) e^{U}\right|\right\rangle_{P} \leq\left\langle e^{M}\left|V_{n}-Y\right|\right\rangle_{P} \\
& =e^{M}\left\langle\left|V_{n}-Y\right|\right\rangle_{P} \rightarrow 0 \text { as } n \rightarrow \infty \text { by (7). }
\end{aligned}
$$

Therefore:

$$
\begin{gathered}
\lim _{n \rightarrow \infty}\left\langle V_{n} e^{U}\right\rangle_{P}=\left\langle Y e^{U}\right\rangle_{P} . \\
\langle Y\rangle_{P_{U}}=[\text { by }(6)]=\lim _{n \rightarrow \infty}\left\langle V_{n}\right\rangle_{P_{U}}=[\text { by Case } 2]=\lim _{n \rightarrow \infty} \frac{\left\langle V_{n} e^{U}\right\rangle_{P}}{\left\langle e^{U}\right\rangle_{P}} \\
=\frac{\lim _{n \rightarrow \infty}\left\langle V_{n} e^{U}\right\rangle_{P}}{\left\langle e^{U}\right\rangle_{P}}=[\text { by }(8)]=\frac{\left\langle Y e^{U}\right\rangle_{P}}{\left\langle e^{U}\right\rangle_{P}} .
\end{gathered}
$$

\section{Theorem 2.3}

Suppose $P$ is a probability measure on $(\Omega, \Sigma), U_{1}$ and $U_{2}$ are bounded random variables on $(\Omega, \Sigma)$ and $U=U_{1}+U_{2}$.

Suppose $P_{1}=P_{U_{1}}$ and $P_{2}=\left(P_{1}\right)_{U_{2}}$ are consecutive Gibbs modifications. Then $P_{2}=P_{U}$.

Proof

Using Lemma 2.2.2), for any event $A \in \Sigma$ we get:

$$
\begin{gathered}
P_{2}(A)=\frac{\left\langle I_{A} e^{U_{2}}\right\rangle_{P_{1}}}{\left\langle e^{U_{2}}\right\rangle_{P_{1}}}=\frac{\frac{\left\langle I_{A} e^{U_{2}} e^{U_{1}}\right\rangle_{P}}{\left\langle e^{U_{1}}\right\rangle_{P}}}{\frac{\left\langle e^{U_{2}} e^{U_{1}}\right\rangle_{P}}{\left\langle e^{U_{1}}\right\rangle_{P}}} \\
=\frac{\left\langle I_{A} e^{U_{2}} e^{U_{1}}\right\rangle_{P}}{\left\langle e^{U_{2}} e^{U_{1}}\right\rangle_{P}}=\frac{\left\langle I_{A} e^{U_{1}+U_{2}}\right\rangle_{P}}{\left\langle e^{U_{1}+U_{2}}\right\rangle_{P}}=P_{U}(A) .
\end{gathered}
$$

\section{Finite Interaction Model}

Denote $\mathbf{0}=(0, \ldots, 0)$, the origin in $\mathbb{Z}^{v}$.

\section{Definition 3.1.}

A finite interaction model with characteristics $\lambda, r$, $\Phi$ (from Definition 1.4) is a sequence $\left(\Lambda, X, U_{\Lambda}, \mathcal{A}_{\Lambda}\right)$ of four objects defined as follows. 
1. $\Lambda=\left\{t \in \mathbb{Z}^{v} \mid\|t-\mathbf{0}\| \leq N\right\}$ for a fixed positive integer $N$. Thus, $\Lambda$ is a cube in the lattice $\mathbb{Z}^{r}$.

2. $X$ is the fixed random field on $(\Omega, \Sigma)$ introduced in Definition 1.1.

3. A function $U_{\Lambda}: \Omega \rightarrow \mathbb{R}$ is called the interaction energy and is defined by the following:

$$
\text { for any } \omega \in \Omega, U_{\Lambda}(\omega)=\sum_{B \in \mathfrak{B}, B \subset \Lambda} \Phi_{B}(\omega),
$$

where the set $\mathfrak{B}$ is defined in Definition 1.4. $U_{\Lambda}(\omega)$ characterizes the energy of configuration $\omega$ in $\Lambda$.

4. Denote $P_{\Lambda}=\left(P_{0}\right)_{U_{\Lambda}}$, the Gibbs modification of the initial probability $P_{0}$ by the interaction energy $U_{\Lambda}$. $\mathcal{A}_{\Lambda}$ is the probability space $\left(\Omega, \Sigma, P_{\Lambda}\right)$.

This ends the Definition 3.1.

\section{Remark 1}

The random variable $U_{\Lambda}$ is bounded because the sum (9) has a finite number of addends and each $\left|\Phi_{B}\right| \leq \lambda^{S(B)}<\lambda$, since $0 \leq \lambda<1$. Therefore Lemma 2.2.1) holds when stated for $P_{\Lambda}$ instead of $P_{U}: P_{\Lambda}$ is a probability measure on $(\Omega, \Sigma)$ and $\mathcal{A}_{\Lambda}$ is indeed a probability space.

\section{Remark 2}

The random field $\left\{X_{t} \mid t \in \mathbb{Z}^{v}\right\}$ is independent on the base probability space $\left(\Omega, \Sigma, P_{0}\right)$ but it may not be independent on the probability space $\left(\Omega, \Sigma, P_{\Lambda}\right)$.

The finite interaction model describes a physical system with many particles represented by points in an integer cube. The random field $X_{t}$ describes some property of the physical system.

The main parameter $\lambda$ from Definition 1.4 is positive and characterizes the temperature of the system: low values of $\lambda$ correspond to high temperatures. Our interaction model describes systems with fairly high temperatures. For $\lambda=0$ the model describes ideal gas.

The interaction model generalizes some well-known models in statistical mechanics (we give details in Sections 5 and 6). In those models the values of random variables $X_{t}$ are bounded. Here we have a more general case when the values of $X_{t}$ are not bounded.

For brevity we denote $\langle\cdot\rangle_{P_{0}}$ as $\langle\cdot\rangle_{0}$.

\section{Lemma 3.2.}

$$
\begin{gathered}
\text { Suppose } t_{1}, \ldots, t_{n} \in \mathbb{Z}^{v} \backslash \Lambda \quad \text { and } \quad a_{1}, \ldots, a_{n} \in \mathbb{R} \text {. Then } \\
P_{\Lambda}\left[\omega\left(t_{1}\right) \leq a_{1}, \ldots, \omega\left(t_{n}\right) \leq a_{n}\right]=P_{0}\left[\omega\left(t_{1}\right) \leq a_{1}, \ldots, \omega\left(t_{n}\right) \leq a_{n}\right] .
\end{gathered}
$$

\section{Proof}

For any $i=1,2, \ldots, n$, denote $A_{i}=\left\{\omega\left(t_{i}\right) \leq a_{i}\right\}$ and denote $A=\bigcap_{i=1}^{n} A_{i}$.

If $t \in \Lambda$ and $a \in \mathbb{R}$, the events $A_{i}$ and $\{\omega(t) \leq a\}$ are independent with respect to the probability measure $P_{0}$, that is on the probability space $\left(\Omega, \Sigma, P_{0}\right)$; this follows from the Definition 1.1. Based on that, similarly to Lemma 1.5 it is proven that for any $B \subset \Lambda$ the random variables $I_{A}$ and $\Phi_{B}$ are independent with respect to $P_{0}$. Therefore $I_{A}$ and $e^{U_{\Lambda}}=\prod_{B \in \mathcal{Z}, B \subset \Lambda} e^{\Phi_{B}}$ are independent with respect to $P_{0}$. Using this independence we get:

$$
\begin{gathered}
P_{\Lambda}\left[\omega\left(t_{1}\right) \leq a_{1}, \ldots, \omega\left(t_{n}\right) \leq a_{n}\right] \\
=P_{\Lambda}(A)=\frac{\left\langle I_{A} e^{U_{\Lambda}}\right\rangle_{0}}{\left\langle e^{U_{\Lambda}}\right\rangle_{0}}=\frac{\left\langle I_{A}\right\rangle_{0}\left\langle e^{U_{\Lambda}}\right\rangle_{0}}{\left\langle e^{U_{\Lambda}}\right\rangle_{0}} \\
=\left\langle I_{A}\right\rangle_{0}=P_{0}(A)=P_{0}\left[\omega\left(t_{1}\right) \leq a_{1}, \ldots, \omega\left(t_{n}\right) \leq a_{n}\right] .
\end{gathered}
$$

For the rest of this section we fix the finite interaction model from Definition 3.1.

\section{Definition 3.3}

Here we introduce random variables $U^{\prime}$ and $U^{\prime \prime}$. For any $\omega \in \Omega$ we define:

$$
U^{\prime}(\omega)=\sum_{B \in \mathfrak{B}, B \subset \Lambda,|B|=1} \Phi_{B}(\omega), U^{\prime \prime}(\omega)=\sum_{B \in \mathfrak{B}, B \subset \Lambda,|B|>1} \Phi_{B}(\omega) .
$$

Thus, $U=U^{\prime}+U^{\prime \prime}$. The function $U$ is split into $U^{\prime}$ and $U^{\prime \prime}$, where $U^{\prime}$ is a sum over singleton sets $B$ and $U^{\prime \prime}$ is a sum over sets $B$ with two or more elements.

Consider consecutive Gibbs modifications $P^{\prime}=\left(P_{0}\right)_{U}$, and $P^{\prime \prime}=\left(P^{\prime}\right)_{U^{\prime \prime}}$. We call $P^{\prime}$ the single modification and $P^{\prime \prime}$ the plural modification.

\section{Lemma 3.4.}

1) For any $t \in \Lambda$ and $x \in \mathbb{R}$ :

$$
P^{\prime}\left(X_{t} \leq x\right)=\frac{\left\langle I_{A} e^{\Phi_{(t)}}\right\rangle_{0}}{\left\langle e^{\Phi_{i t}}\right\rangle_{0}},
$$

where $A=\left\{X_{t} \leq x\right\}$.

2) For any $t \in \mathbb{Z}^{v} \backslash \Lambda$ and $x \in \mathbb{R}$ :

$$
P^{\prime}\left(X_{t} \leq x\right)=P_{0}\left(X_{t} \leq x\right) .
$$


3) After the single modification the field $\left\{X_{t} \mid t \in \mathbb{Z}^{v}\right\}$ is still independent. That is, this field is independent on the probability space $\left(\Omega, \Sigma, P^{\prime}\right)$.

\section{Proof}

For brevity we will write $\Phi_{\{\tau\}}$ as $\Phi_{\tau}$. Thus:

$U^{\prime}=\sum_{\tau \in \Lambda} \Phi_{\tau}$ and $e^{U^{\prime}}=\prod_{\tau \in \Lambda} e^{\Phi_{\tau}}$

1) $P^{\prime}\left(X_{t} \leq x\right)=P^{\prime}(A)=\frac{\left\langle I_{A} e^{U^{\prime}}\right\rangle_{0}}{\left\langle e^{U^{\prime}}\right\rangle_{0}}=[b y(10)]=\frac{\left\langle I_{A} \prod_{\tau \in \Lambda} e^{\Phi_{\tau}}\right\rangle_{0}}{\left\langle\prod_{\tau \in \Lambda} e^{\Phi_{\tau}}\right\rangle_{0}}$

$=\frac{\left\langle\left(I_{A} \cdot e^{\Phi_{t}}\right) \cdot\left(\prod_{\tau \in \Lambda, \tau \neq t} e^{\Phi_{\tau}}\right)\right\rangle_{0}}{\left\langle e^{\Phi_{t}} \prod_{\tau \in \Lambda, \tau \neq t} e^{\Phi_{\tau}}\right\rangle_{0}}=$ (due to independence with

respect to measure $P_{0}$ by Lemma 1.5)

$$
=\frac{\left\langle I_{A} \cdot e^{\Phi_{t}}\right\rangle_{0} \cdot\left\langle\prod_{\tau \in \Lambda, \tau \neq t} e^{\Phi_{\tau}}\right\rangle_{0}}{\left\langle e^{\Phi_{t}}\right\rangle_{0} \cdot\left\langle\prod_{\tau \in \Lambda, \tau \neq t} e^{\Phi_{\tau}}\right\rangle_{0}}=\frac{\left\langle I_{A} e^{\Phi_{t}}\right\rangle_{0}}{\left\langle e^{\Phi_{t}}\right\rangle_{0}} .
$$

2) Denote $A=\left\{X_{t} \leq x\right\}$.

$P^{\prime}\left(X_{t} \leq x\right)=P^{\prime}(A)=\frac{\left\langle I_{A} e^{U^{\prime}}\right\rangle_{0}}{\left\langle e^{U^{\prime}}\right\rangle_{0}}=[$ by $(10)]=\frac{\left\langle I_{A} \prod_{\tau \in \Lambda} e^{\Phi \tau}\right\rangle_{0}}{\left\langle\prod_{\tau \in \Lambda} e^{\Phi \tau}\right\rangle_{0}}=$ (due to independence with respect to measure $P_{0}$ ) $=$

$$
=\frac{\left\langle I_{A}\right\rangle_{0}\left\langle\prod_{\tau \in \Lambda} e^{\Phi \tau}\right\rangle_{0}}{\left\langle\prod_{\tau \in \Lambda} e^{\Phi \tau}\right\rangle_{0}}=\left\langle I_{A}\right\rangle_{0}=P_{0}(A)=P_{0}\left(X_{t} \leq x\right) \text {. }
$$

3) We need to prove that for any $a_{1}, \ldots, a_{n} \in \mathbb{R}$ and distinct $t_{1}, \ldots, t_{n} \in \mathbb{Z}^{v}$ :

$$
\begin{aligned}
& P^{\prime}\left(X_{t_{1}} \leq a_{1}, X_{t_{2}} \leq a_{2}, \ldots, X_{t_{n}} \leq a_{n}\right) \\
& =P^{\prime}\left(X_{t_{1}} \leq a_{1}\right) \cdot \ldots \cdot P^{\prime}\left(X_{t_{n}} \leq a_{n}\right) .
\end{aligned}
$$

Denote $t=t_{1}, B=\left\{X_{t} \leq a_{1}\right\}$ and $C=\left\{X_{t_{2}} \leq a_{2}, \ldots, X_{t_{n}} \leq a_{n}\right\}$. First we prove:

$$
P^{\prime}(B \cap C)=P^{\prime}(B) P^{\prime}(C) .
$$

Its left-hand side equals:

$$
P^{\prime}(B \cap C)=\frac{\left\langle I_{B \cap C} e^{U^{\prime}}\right\rangle_{0}}{\left\langle e^{U^{\prime}}\right\rangle_{0}}=[b y(10)]=\frac{\left\langle I_{B} \cdot I_{C} \prod_{\tau \in \Lambda} e^{\Phi_{\tau}}\right\rangle_{0}}{\left\langle\prod_{\tau \in \Lambda} e^{\Phi_{\tau}}\right\rangle_{0}} .
$$

Consider 2 cases.

- $\quad$ Case 1: $t \in \Lambda$.

Then:

$$
P^{\prime}(B \cap C)=\frac{\left\langle\left(I_{B} \cdot e^{\Phi_{t}}\right) \cdot\left(I_{C} \prod_{\tau \in \Lambda, \tau \neq t} e^{\Phi_{\tau}}\right)\right\rangle_{0}}{\left\langle e^{\Phi_{t}} \prod_{\tau \in \Lambda, \tau \neq t} e^{\Phi_{\tau}}\right\rangle_{0}}
$$

$=\left(\right.$ due to independence with respect to measure $P_{0}$ by Lemma 1.5):

$$
=\frac{\left\langle I_{B} \cdot e^{\Phi_{t}}\right\rangle_{0} \cdot\left\langle I_{C} \prod_{\tau \in \Lambda, \tau \neq t} e^{\Phi_{\tau}}\right\rangle_{0}}{\left\langle e^{\Phi_{t}}\right\rangle_{0}\left\langle\prod_{\tau \in \Lambda, \tau \neq t} e^{\Phi_{\tau}}\right\rangle_{0}}=\frac{\left\langle I_{B} \cdot e^{\Phi_{t}}\right\rangle_{0}}{\left\langle e^{\Phi_{t}}\right\rangle_{0}} \cdot \frac{\left\langle I_{C} \prod_{\tau \in \Lambda, \tau \neq t} e^{\Phi_{\tau}}\right\rangle_{0}}{\left\langle\prod_{\tau \in \Lambda, \tau \neq t} e^{\Phi_{\tau}}\right\rangle_{0}} \cdot \frac{\left\langle e^{\Phi_{t}}\right\rangle_{0}}{\left\langle e^{\Phi_{t}}\right\rangle_{0}}
$$

$=[$ by part 1$)]$

$$
=P^{\prime}(B) \cdot \frac{\left\langle I_{C} \prod_{\tau \in \Lambda} e^{\Phi_{\tau}}\right\rangle_{0}}{\left\langle\prod_{\tau \in \Lambda} e^{\Phi_{\tau}}\right\rangle_{0}}=P^{\prime}(B) \cdot \frac{\left\langle I_{C} e^{U^{\prime}}\right\rangle_{0}}{\left\langle e^{U^{\prime}}\right\rangle_{0}}=P^{\prime}(B) \cdot P^{\prime}(C) .
$$

- Case 2: $t \in \mathbb{Z}^{v} \backslash \Lambda$.

Due to independence with respect to measure $P_{0}$ we have:

$$
\begin{aligned}
P^{\prime}(B \cap C) & =\frac{\left\langle I_{B} \cdot I_{C} \prod_{\tau \in \Lambda} e^{\Phi_{\tau}}\right\rangle_{0}}{\left\langle\prod_{\tau \in \Lambda} e^{\Phi_{\tau}}\right\rangle_{0}}=\frac{\left\langle I_{B}\right\rangle_{0}\left\langle I_{C} \prod_{\tau \in \Lambda} e^{\Phi_{\tau}}\right\rangle_{0}}{\left\langle\prod_{\tau \in \Lambda} e^{\Phi_{\tau}}\right\rangle_{0}} \\
= & \left\langle I_{B}\right\rangle_{0} \frac{\left\langle I_{C} \prod_{\tau \in \Lambda} e^{\Phi_{\tau}}\right\rangle_{0}}{\left\langle\prod_{\tau \in \Lambda} e^{\Phi_{\tau}}\right\rangle_{0}}=P_{0}(B) P^{\prime}(C)=P^{\prime}(B) P^{\prime}(C)
\end{aligned}
$$

by part 2). Thus, (12) is proven. Then:

$$
\begin{aligned}
& P^{\prime}\left(X_{t_{1}} \leq a_{1}, X_{t_{2}} \leq a_{2}, \ldots, X_{t_{n}} \leq a_{n}\right) \\
& =P^{\prime}\left(X_{t_{1}} \leq a_{1}\right) \cdot P^{\prime}\left(X_{t_{2}} \leq a_{2}, \ldots, X_{t_{n}} \leq a_{n}\right) .
\end{aligned}
$$

Continuing by induction we get (11). 
Thus, the single modification can change distributions of random variables $X_{t}\left(t \in \mathbb{Z}^{v}\right)$ but it does not change their independence. Note that we do not put any restriction on initial distributions of $X_{t}$.

\section{Infinite Interaction Model}

\section{Definition 4.1}

An infinite interaction model with characteristics $\lambda$, $r, \Phi$ is the ordered sequence of two objects $(X, \mathcal{A})$ defined as follows.

1. $X$ is the fixed random field on $(\Omega, \Sigma)$ introduced in Definition 1.1.

2. For any $N=1,2, \ldots$ denote $\Lambda_{N}=\left\{t \in \mathbb{Z}^{v} \mid\|t-\mathbf{0}\| \leq N\right\}$.

Let $\left(\Lambda_{N}, X_{\Lambda_{v}} \mathcal{A}_{\Lambda_{v}}\right)$ be the finite interaction model and $P_{N}=P_{\Lambda_{N}}$ the corresponding probability measure from Definition 3.1.

Let $P_{\lambda}$ be a probability measure on $(\Omega, \Sigma)$ that is a limit of the probability measures $P_{N}$ in some sense, for example, their weak limit, as $N \rightarrow \infty$.

$\mathcal{A}$ is defined by:

$$
\mathcal{A}=\left(\Omega, \Sigma, P_{\lambda}\right) .
$$

Thus, $\mathcal{A}$ is a probability space.

3. The probability measure $P_{\lambda}$ is called Gibbs measure.

4. The infinite interaction model $(X, \mathcal{A})$ is also called the thermodynamic limit or macroscopic limit of the finite interaction models $\left(\Lambda_{N}, X, U_{\Lambda_{N}} \mathcal{A}_{\Lambda_{N}}\right)$ as $N \rightarrow \infty$.

\section{Remark}

The problem of existence of the probability measure $P_{\lambda}$ as a limit of the probability measures $P_{N}$ is studied in literature but not in a rigorous mathematical context; it needs further investigation.

\section{Particular Cases of Interaction Model}

\section{Ising Model}

Ising model is an important mathematical model of ferromagnetism in statistical mechanics. It can be described as a particular case of the interaction model:

- each configuration $\omega: \mathbb{Z}^{v} \rightarrow\{ \pm 1\}$;

- $P_{0}\left(X_{s}=1\right)=P_{0}\left(X_{s}=-1\right)=0.5$;

- $r=1$;
- $\Phi_{\{s\}}(\omega)=\lambda h_{s} X_{s}(\omega)$, where $h_{s}\left(s \in \mathbb{Z}^{v}\right)$ are real numbers characterizing influence of an external field;

- $\Phi_{\{s, t\}}(\omega)=\lambda J_{s t} X_{s}(\omega) X_{t}(\omega)$ if $\|s-t\|=1$; here $J_{s t}$ are real numbers characterizing interaction of points $s$ and $t$;

- $\quad$ in other cases $\Phi_{B}=0$.

According to Definition 3.3, $U_{\Lambda}=U^{\prime}+U^{\prime \prime}$, where:

$$
\begin{aligned}
& U^{\prime}(\omega)=\sum_{t \in \Lambda} h_{t} X_{t}(\omega), \\
& U^{\prime \prime}(\omega)=\sum_{s, t \in \Lambda} \lambda J_{s t} X_{s}(\omega) X_{t}(\omega) .
\end{aligned}
$$

After the single modification (by $U^{\prime}$ ) the distribution of $X_{t}$ is still independent and is given by:

$$
P^{\prime}\left(X_{t}=-1\right)=\frac{e^{-h_{t}}}{e^{-h_{t}}+e^{h_{t}}}=\frac{1}{1+e^{2 h_{t}}} ; P^{\prime}\left(X_{t}=1\right)=\frac{e^{2 h_{t}}}{1+e^{2 h_{t}}} .
$$

After the plural modification (by $U^{\prime \prime}$ ) we get the Ising model, that is the modification by $U_{\Lambda}$, as proven in Theorem 2.3. Modification in two steps can simplify computations.

Some authors study the Ising model with four point interaction; see, for example, (Yang et al., 2017) and references in it. In that case we add to the above definition of $\Phi_{B}$ a clause for a 4-point set $B$ :

$$
\Phi_{B}(\omega)=\lambda J_{B} \prod_{t \in B} X_{t}(\omega),
$$

where $B$ consists of the vertices of a unit square or the vertices of a tetrahedron with three edges of length 1 and three other edges of length $\sqrt{2}$.

\section{Potts Model}

Standard Potts model can be described as a particular case of the interaction model:

- each configuration $\omega: \mathbb{Z}^{v} \rightarrow\{1,2, \ldots, q\}$;

- $P_{0}\left(X_{t}=i\right)=\frac{1}{q}, i=1,2, \ldots, q$;

- $r=1$;

- $\Phi_{\{s, t\}}(\omega)=\lambda J_{s t} \delta\left(X_{s}(\omega), X_{t}(\omega)\right)$ if $\|s-t\|=1$; here $\delta(x, y)= \begin{cases}1 & \text { if } x=y, \\ 0 & \text { if } x \neq y .\end{cases}$

In other cases $\Phi_{B}=0$.

The Ising model is a particular case of the standard Potts model when $q=2$ (it can be reduced to the Ising model by linear transformation $X_{t} \rightarrow 2 X_{t}-3$ ). 


\section{Discussion}

In this study we develop a mathematically rigorous concept of interaction model for a physical system with many particles, which interact with an external force and with one another; a random field $X_{t}\left(t \in \mathbb{Z}^{v}\right)$ models some property of the system such as electric charge, density etc. We introduce a finite model first and then define the thermodynamic limit of the finite models with Gibbs probability measure. Unlike most existing models, in our model the set of values of $X_{t}$ can be unbounded, which provides more generality.

We study properties of the interaction model. In particular, we split Gibbs modification of the initial independent probability measure into two steps reflecting the influence of an external field on the first step and the interaction between particles on the second step. The first-step modification is mathematically simple and leaves the field $X_{t}$ independent, therefore this construction simplifies mathematical computations.

Next we show that Ising and Potts models are particular cases of the interaction model. If we change the set of values of the random field $X_{t}$ from $\mathbb{R}$ to $\mathbb{R}^{n}$, then the generalized interaction model will also include the $n$-vector model and its special cases for $n=2$ ( $X Y$-model) and $n=3$ (Heisenberg model). We are planning to research further mathematical properties and physical applications of the interaction model.

\section{Acknowledgement}

The authors thank the journal's Editor in Chief for his valuable support of this article.

\section{Author's Contributions}

Farida Kachapova and Ilias Kachapov: Contributed to the preparation, development and publication of the manuscript.

\section{Ethics}

This is a mathematical article; no ethical issues can arise after its publication.

\section{References}

Duminil-Copin, H., R. Peled, W. Samotij and Y. Spinka, 2017. Exponential decay of loop lengths in the loop $O(n)$ model with large $n$. Commun. Math. Phys., 349: 777-817. DOI: 10.1007/s00220-016-2815-4.

Kachapova, F. and I. Kachapov, 2016. Convergence of renormalization group transformations of Gibbs random field. J. Math. Statist., 12: 135-151. DOI: 10.3844/jmssp.2016.135.151.

Kashapov, I.A., 1977. Structure of ground states in three-dimensional Ising model with three-step interaction. Theor. Math. Phys., 33: 110-118.

Külske, C.K., U.A. Rozikov and R.M. Khakimov, 2014. Description of the translation-invariant splitting Gibbs measures for the Potts model on a Cayley tree. J. Statist. Phys., 156: 189-200. DOI: 10.1007/s10955-014-0986-y.

Malyshev, V.A. and R.A. Minlos, 1991. Gibbs Random Fields: Cluster Expansions. 1st Edn., Kluwer Academic, Dordrecht, Netherlands, ISBN-10: 079230232X, pp: 248.

Malyshev, V.A., 1980. Cluster expansions in lattice models of statistical physics and the quantum theory of fields. Russian Math. Surveys, 35: 1-62.

Yang, Y., B. Teng, H. Yang and H. Cui, 2017. Investigation of probability theory on Ising models with different four-spin interactions. Physica A: Statist. Mech. Applied, 483: 243-249. DOI: 10.1016/j.physa.2017.04.176. 